Brigitte Leal Vásquez

\author{
Abogada asistente, Tribunal Constitucional \\ Santiago, Chile \\ bleal@tcchile.cl
}

\title{
Ciencia y Derecho: La nueva división de Poderes José Esteve Pardo y Javier Tejada Palacios Fundación Coloquio Jurídico Europeo, 2013, 236páginas
}

\section{Introducción}

La obra que vamos a comentar se enmarca dentro de la progresiva línea de investigación que el Prof. Dr. José Esteve Pardo ha ido desarrollando en los últimos años sobre el concepto de riesgo y su gestión por el Derecho Administrativo. Dicho eje de estudio ha sido expuesto en varias de sus obras. Baste con nombrar, por ejemplo, "Técnica, riesgo y derecho" (1999), "Autorregulación. Génesis y Efectos" (2001), "El desconcierto del Leviatán. Política y Derecho ante las incertidumbres de la ciencia” (2009) y sus "Lecciones de Derecho Administrativo" (quinta edición, 2015), manual en el cual le otorga un capítulo especial y desde el cual es posible apreciar el encuadre general que el autor le atribuye al riesgo dentro del estudio de esta rama del Derecho.

Primero, es importante señalar ciertas cosas sobre los autores. Por un lado, el Prof. Dr. José Esteve Pardo es catedrático de Derecho Administrativo en la Universidad de Barcelona. Su destacada carrera académica lo ha llevado a ser profesor visitante en innumerables universidades extranjeras, de entre las que destacan la Universidad Munich y la Northwestern University. Ha formado parte, además, de importantes consejos consultivos, como el Consejo de Redacción de la revista European Journal of Risk Regulation. Se ha consolidado dentro del escenario jurídico-dogmático como vasto conocedor de las materias que forman parte de la regulación pública actual.

Por otro lado, Javier Tejada Palacios es catedrático de Física de la Univerdad de Barcelona, desempeñándose además en importantes actividades de investigación. Ha recibido diversos premios y reconocimientos en su ámbito, como el Premio Príncipe de Viana de la Cultura Y el Premio Nacional de Física. Además, ha sido nombrado Doctor Honoris Causa de la City University of New York. Ha publicado variadas obras, dentro de las cuales cabe destacar "El Templo de la Ciencia" (2008) y "El viaje de Cloe" (2011). 


\section{La obra}

La presente obra es fruto de las ponencias que los autores realizaron en el coloquio $\mathrm{N}^{\circ} 45$ de la Fundación Coloquio Jurídico Europeo, en Madrid, el año 2012. En ella, el autor principal es el Prof. Dr. Esteve Pardo, seguido del profesor Javier Tejada Palacios, quien realiza un ensayo más breve hacia el final de la obra, abordando el tema desde la perspectiva de la ciencia.

La idea central de la obra - y con la cual comienza su exposición el Prof. Dr. Esteve Pardo - es la configuración de la ciencia como un nuevo poder, nacido al margen de la clásica división tripartita erigida por la ciencia política hace ya más de doscientos años. Su surgimiento, fuera del seno del Estado, constituye uno de sus elementos diferenciadores y el cual ha venido a caracterizar las nuevas relaciones entre el Estado y los ciudadanos.

La ciencia como poder constituye un fenómeno social en aumento. Buena parte del devenir y del bienestar social es atribuido, mayoritariamente, al progreso técnico y científico. Por otra parte, su importancia para los gobiernos no deja de ser menor, quienes han establecido amplias y potentes agendas para plantear los actuales dilemas de la ciencia y cómo estos constituyen, en no pocas ocasiones, la variable principal a la hora de tomar decisiones.

En dicho contexto, la legitimidad del juicio experto ha cobrado una importancia que hasta hace pocos años era desconocida. No son pocas las ocasiones en que el discurso de la ciencia logra relegar al derecho, dejándolo en un segundo plano, al momento de determinar los criterios que fundamentan la decisión de la autoridad. Aún más, en muchos otros casos, el veredicto de la ciencia se torna decisivo y se erige como única opción posible, lo cual es especialmente importante en contextos donde el derecho no puede excusarse de tomar una decisión. En todos ellos, la influencia de la ciencia y, específicamente, de la tecnociencia (aquel complejo entramado existente entre la ciencia y el desarrollo tecnológico), puede resultar decisivo para el devenir de las sociedades actuales. Desde este punto, el autor plantea los nuevos escenarios en los cuales se desarrolla la relación entre el derecho y la ciencia y cómo esta última ha reconfigurado tanto estructural como funcionalmente al primero.

La obra consta de siete capítulos, cada uno de los cuales desarrolla por sí solo un tema en específico que sirve de apoyo para las ideas principales que el autor afirma hacia los últimos capítulos de la misma: una crítica a la deriva cientificista del derecho.

En primer lugar, el autor nos ilustra sobre la expansión que ha tenido la incertidumbre en las sociedades postmodernas. Ello ha significado un verdadero reto para el derecho cuando debe decidir, invariablemente, en situaciones de incerteza, ya que la capacidad de la ciencia para generar certezas y contribuir a la toma de decisiones es cada vez menor. Sus veredictos ya no son categóricos, estando dominados esencialmente por el lenguaje de la probabilidad. De esta manera, su actual aporte al derecho es, querámoslo o no, más bien humilde.

Para los gobernantes, decidir en contextos de incertidumbre se ha vuelto, a lo menos, una cuestión cotidiana. El impacto del cambio climático en el medio ambiente, el potencial efecto nocivo de la existencia de ondas electromagnéticas, la liberación de organismos genéticamente modificados, entre otros casos, ejemplifican esta situación. En todos ellos, la ciencia no ha decidido, lo ha hecho el derecho: la Unión Europea, los organismos internacionales, los gobiernos centrales, las autoridades locales, los tribunales 
de justicia, etc., ya sea de manera afirmativa o negativa - o incluso por la vía del silencio - frente a la ocurrencia de riesgos ambientales, sanitarios, económicos o sociales ${ }^{1}$.

De esta manera, el autor llega a uno de los puntos centrales de su obra: la nueva correlación de fuerzas existente entre la ciencia y el derecho ${ }^{2}$. Esta relación ha sido caracterizada por la importancia del progreso científico y tecnológico, el cual ha permitido decidir en ámbitos donde hasta hace no mucho tiempo atrás reinaba el devenir propio de los procesos naturales, al margen de toda intervención humana ${ }^{3}$. A su juicio, las manifestaciones más importantes de esta nueva relación son: una sociedad enfrentada constantemente al riesgo tecnológico, una apertura reflexiva hacia la investigación científica y su aplicación tecnológica y el mayor conocimiento obtenido sobre los procesos naturales que el poder debe dominar o evitar, como la emisión de gases a la atmósfera, la afectación de la capa de ozono, el peligro que acarrean los huracanes, etc. En todas estas manifestaciones, el componente natural es desplazado por el protagonismo de la tecnología, siendo los gobiernos, en conjunto con las industrias, los llamados a controlar y a rediseñar la gestión de este tipo de riesgos.

Lo anterior conlleva, por cierto, la expansión de la regulación del derecho a espacios hasta antes impensados. De la misma manera, crece también la responsabilidad que pesa sobre la decisión de la autoridad en cada uno de estos casos ${ }^{4}$. Por suerte, el autor es bastante directo al advertir que, derechamente, estamos en presencia de un problema precedido por importantes consideraciones políticas ${ }^{5}$, las que hasta el momento han favorecido el progreso científico, mejorando condiciones de vida, el desarrollo de la sociedad moderna y el incremento de la industrialización sostenible.

Esta responsabilidad que existe en la decisión humana es creciente. No obstante, al momento de constatar que ese grupo de decisiones puede conllevar efectos negativos en diversos aspectos, como el sanitario, el ambiental, el económico, etc., el consenso sobre el beneficio del progreso científico y tecnológico se rompe, dando paso a miradas más bien preventivas hacia la técnica y sus riesgos ${ }^{6}$.

De esta manera, el autor abre paso hacia el siguiente gran tema que plantea en su obra: la incertidumbre en los nuevos espacios de decisión y los problemas que ello genera para el derecho. Porque, como es destacado en su obra, el desarrollo tecnológico no sólo ha solventado soluciones importantes en la vida de las personas, sino que también ha creado problemas no menores, bajo los cuales las posiciones más refractarias se han escudado. El deterioro del medio ambiente, los efectos sobre el clima y, en general, el riesgo inherente del progreso científico y técnico sostienen un rechazo o, al menos, una postura moderada, hacia la promoción de la técnica. El epítome de ello se observa en aquellos casos en donde es el propio ser humano - y no su entorno natural - el objeto directo de la técnica, cuestión desarrollada por la biogenética y la biotecnología ${ }^{7-8}$.

\footnotetext{
Esteve Pardo, José; Tejada Palacios, Javier, Ciencia y Derecho: La nueva división de Poderes, Fundación Coloquio Jurídico Europeo, 2013, pp. $28-30$.

Íbid., p. 32.

Íbid., p. 32.

Íbid., pp. $34-36$

Íbid., p. 38.

Íbid., p. 44 .

Íbid., p. 46.

Íbid., p. 47.
} 
Como sostiene el autor, los problemas para el derecho que plantea el desarrollo de la ciencia y la técnica son insospechadamente importantes. Ello es así por cuanto muchas de las premisas sobre las cuales el derecho transita están basadas en el principio de seguridad jurídica, el cual colisiona, indefectiblemente, con la incertidumbre planteada por la ciencia?.

Para el derecho, señala el autor, la certeza es uno de sus bienes más preciados. En ella se basa, de hecho, el sistema jurídico ${ }^{10}$. Dicha aspiración de certeza se originó, siglos atrás, con el auge de las ciencias experimentales. Las leyes de Newton - ejemplifica el autor - ejercieron una influencia decisiva en el pensamiento filosófico y político de Kant y, como sabemos, su influencia se extiendió casi de manera completa a la construcción del sistema jurídico y constitucional sobre el cual aún hoy nos movemos ${ }^{11}$. Sin embargo, con el paso del tiempo, la ciencia cambió de rumbo. Dejó de ser aquella área formadora de certezas y, por el contrario, pasó a proveer al mundo de innumerables juicios probabilísticos. Hacia finales del siglo XX, la certeza ya no era ni siquiera un objetivo de la ciencia, y muchas de las previamente establecidas comenzaron a desvanecerse. Con ello, "el fin de las certidumbres" ${ }^{12}$ arribó y, junto con él, el nuevo y actual paradigma de intervención del derecho en la sociedad.

De acuerdo al autor, las respuestas del derecho a este nuevo desafío han sido variadas. Desde luego, muchas de ellas han exigido salir del positivismo, ya que buscar las herramientas necesarias para controlar los riesgos ha supuesto, sobre todo, una mirada interdisciplinaria. El principio de precaución es sólo el caso más paradigmático. Pero sin duda hay más. Por ello, una teoría de la decisión jurídica en contextos de incertidumbre se ha ido modelando de manera lenta y silenciosa, siendo urgente que la sociedad entera reflexione en torno a ello ${ }^{13}$.

Luego, la pregunta sobre el sujeto de la decisión, esto es, “¿decide la ciencia o decide el derecho?” representa el núcleo esencial de los esfuerzos argumentativos en la parte central de su obra. La respuesta, a estas alturas, resulta evidente. Sin embargo, lo más importante - de acuerdo al autor - es determinar las condiciones bajo las cuales los órganos políticos ejercen ese poder de decisión ${ }^{14}$.

Desde sus diferentes plataformas de legitimación, el derecho y la ciencia se entrecruzan en infinitas situaciones de toma de decisiones. Situaciones no menos tensas, por cierto, ya que en muchos de esos casos, la ciencia acaba por rebasar los límites impuestos por el poder del derecho, trasladando la autoridad de su conocimiento a las instancias de decisión jurídico-públicas. Ejemplos de estas "vías de entrada de la ciencia y la técnica en el espacio público de decisión" son variados ${ }^{15}$. El autor repasa varios de ellos, como la previa determinación que debe realizar la ciencia para que el derecho arribe a una solución eficiente (como la precisión del nivel de contaminación que pone en peligro la salud humana); el poder de la tecnocracia y expertocracia en los espacios de regulación y decisión jurídica (como el auge de la autorregulación y los organismos de normalización); la remisión que el derecho realiza a las soluciones dadas por la ciencia (las llamadas "cláusulas técnicas"); y la toma de poder decisorio por parte de la ciencia

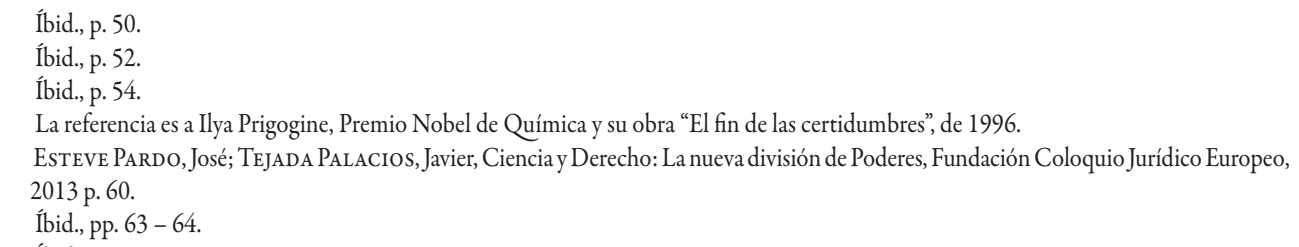


ante la superación del derecho en virtud de la velocidad de sus avances (como el caso de los embriones sobrantes de tratamientos realizados por la tecnología biogenética $)^{16}$.

Teniendo en consideración esta expansión de la ciencia sobre el derecho, el autor plantea su tesis de manera categórica: el derecho debe recuperar sus espacios propios de decisión, restaurando sus valores y sus marcos de referencia. Ello significa retomar el rumbo que la ciencia - con su autoridad y hegemonía - le ha desfigurado. Para ello, el autor aduce sus razones. La primera dice relación con la legitimación constitucional que las instancias de decisión del derecho poseen. La intervención en la comunidad política debe tener el grado más alto de validación, cuestión que es alcanzada por medio de los mecanismos formales de producción de decisiones en el ámbito del derecho y no en el de la ciencia. La segunda dice relación con la vigencia del principio democrático, en donde los sistemas de decisión del derecho sí poseen la capacidad de involucrar a una comunidad que decide sobre cuestiones que efectivamente le afectan. Finalmente, porque la decisión - aunque en sustancia contenga designios propios de la ciencia - en la mayoría de los casos es imputada a un órgano jurídico y no a uno de carácter científico, y en ellos se radican las consecuencias de dichas decisiones ${ }^{17}$.

Para fortalecer su postura en torno a la recuperación de los espacios de decisión propios del derecho, el autor plantea la discusión en torno a una reserva de decisión política y regulación jurídica en sectores claves del desarrollo tecnológico. De esta manera, ciertos ámbitos de la técnica deben ser decididos por entes dotados de legitimidad democrática ${ }^{18}$. Evidentemente, resulta conveniente no radicar - al menos no del todo - este grupo de materias en el parlamento. Los necesarios elementos políticos que existen detrás de las mismas podrían trabar el buen puerto de las soluciones requeridas, muchas de las cuales son de carácter urgente. Lo que sí necesariamente debe decidir el parlamento, de acuerdo al autor, es el "organigrama", esto es, la configuración de los órganos llamados a tomar este tipo de decisiones, entendiéndolos de un modo que asegure la independencia, la representatividad y el conocimiento científico ${ }^{19}$. En esta labor, el derecho no puede seguir claudicando, debiendo enfrentar a la ciencia y no rendirse ante ella, mediante parámetros y marcos de referencia que potencien su apoyo a los órganos públicos llamados a decidir ${ }^{20}$.

Llegamos así al penúltimo capítulo de la obra, donde se analiza la aplicación del principio de precaución como elemento articulador de las relaciones entre la ciencia y el derecho en situaciones de incertidumbre, ejemplificando de esta manera la deriva cientificista del derecho ${ }^{21}$. De dicho principio, lo más destacado por el autor son las consecuencias de su aplicación, refiriéndose a ellas como un "estado de excepción declarado por la ciencia” puesto que las medidas que se derivan de su aplicación, en muchas ocasiones, excepcionan el régimen jurídico vigente, creando - en algunos casos - verdades situaciones de anomia ${ }^{22}$.

Desde las críticas que ha suscitado la aplicación del principio de precaución, el autor aborda - en el capítulo final de su obra -la reivindicación de los modelos de decisión característicos del derecho. Estas críticas son: en primer lugar, una remisión irreflexiva a la ciencia y a la tecnología; en segundo lugar, la neutralización del derecho y el aumento de decisiones de contenido negativo; en tercer lugar, la irres-

Íbid., pp. $66-81$

Íbid., pp. $82-84$.

Íbid., p. 87.

Íbid., p, 88 .

Íbid., p. 91.

Íbid., p.123.

Íbid., p.130. 
ponsabilidad de la ciencia ante medidas injustificadas infundadas de reacción frente a riesgos ${ }^{23}$. Está claro que estos conjuntos de disfunciones no pueden fundar una fe ciega en las formas jurídicas y en el inmovilismo de las decisiones fundadas en patrones normativos. En el balance - señala el autor - está la clave a la hora de establecer los mecanismos y modelos de decisión aptos para enfrentar las situaciones de incertidumbre ${ }^{24}$.

No cabe duda que el espacio construido por las certezas del derecho suele no coincidir con el espacio real. $\mathrm{Al}$ reconstruir la realidad, el derecho se sitúa un paso más cerca del relato propio de la literatura que del de la ciencia y su pretensión de objetividad ${ }^{25}$. La certeza aquí no es certeza, es seguridad jurídica. Y, para arribar a ella, están los procedimientos. De ahí que cada uno de los presupuestos formales y previos a una decisión, en cualquier contexto, sean determinantes. Porque, al contrario de la ciencia, lo importante para el derecho será, en general, el cumplimiento de estos requisitos. Pocas veces lo será el resultado ${ }^{26}$. Y cuando esto último ocurre, esto es, cuando el derecho sí posee un compromiso con la materialidad de la decisión, se arribará a ella en virtud de valores superiores que propendan hacia la búsqueda de una verdad empírica. En el ámbito penal, lo anterior resulta ser particularmente, al proscribirse la prueba ilícita, pero siendo necesario, para condenar una prueba más allá de toda duda razonable que rompa con la situación previa de presunción de inocencia. En esto último, no caben situaciones de incertidumbres ${ }^{27}$.

De esta manera, el autor comienza a cerrar su exposición con algunas conclusiones. En primer lugar, señala que el conocimiento, por muy fundado o científicamente comprobado que sea, no es un valor absoluto para el sistema de decisiones liderado por el derecho. De ahí que casos emblemáticos, como la prueba ilícita, sean jurídicamente reprochables en un mayor o menos sentido. En segundo lugar, la forma literaria (de recreaciones de la realidad) y no científica del derecho explica el rol preponderante de las ficciones, cuando la certeza y la seguridad jurídica suponen un valor público superior ${ }^{28}$.

Respecto de la segunda conclusión, el autor menciona que las ficciones del derecho, establecidas típicamente a través de las presunciones, pueden ser útiles para enfrentar los casos de incertidumbre cuando incluyen una valoración del legislador frente a la misma. Si, tradicionalmente, esa valoración no era más que la expresión de las máximas de experiencia de una época y tiempo dados, hoy pueden ir más allá de la experiencia, de la estadística y de lo comprobable. Incluye, de hecho, una potenciación y protección de un bien jurídico o de una posición que podría verse afectada por la situación de incertidumbre a enfrentar, como la dificultad de probar ciertos hechos en virtud de su alta complejidad técnicaa ${ }^{29}$ o en aquellos casos donde es necesaria una consideración de equidad ${ }^{30}$.

Finalmente, el autor aborda lo que ha llamado las "presunciones institucionales de conocimiento científico", donde el legislador establece una presunción de autoridad a favor de una entidad científica determinada, como ocurre con las agencias de seguridad farmacológica, de inocuidad alimentaria, de seguridad nuclear, ambientales, entre otras. En todos estos casos, la presunción institucional de conoci-

\footnotetext{
Íbid., 143-145.

Íbid., p. 146.

Íbid., p. 150.

Íbid., p. 153.

Íbid., p. 159.

Íbid., p. 165.

Íbid., p. 176.

Íbid., p. 179.
} 
miento científico atribuye una importante influencia a estas entidades en la decisión que una determinada entidad políticamente legitimada deba tomar en contextos de incertidumbre, superando cualquier controversia paralizante dentro de la comunidad científica. Y si bien el autor no espera en advertir que esta presunción podría significar un retorno a la deriva cientificista del derecho, especifica que, en este caso, existe una previa decisión por parte del derecho de determinar la agencia específica que será la única fuente de información relevante para que la entidad política correspondiente tome una decisión ${ }^{31}$.

\section{La perspectiva desde el "Templo de la Ciencia"}

De esta manera, y tras el elogiable esfuerzo de pasar revista a importantes temas de la teoría del derecho y, en particular, del Derecho Público, la obra se cierra con el ensayo de Javier Tejada Palacios quien, desde la óptica de las ciencias experimentales, expone su visión sobre esta nueva división de poderes entre la ciencia y el derecho. Principalmente, discurre en torno a cómo la ciencia y la tecnología no solo han introducido incertidumbres en el derecho sino (mucho más grave para él) en el propio "Templo de la Ciencia”, del cual solo conocemos una minúscula parte ${ }^{32}$.

De su ensayo, más breve que el anterior, existen dos ideas fundamentales a destacar. En primer lugar, su premisa fundamental, cual es, que el conocimiento científico está en permanente evolución y que cada nuevo descubrimiento dentro de él significa cambiar la visión de prácticamente todo lo ya existente. Lo anterior, de acuerdo al autor, dificulta las explicaciones que satisfacen la búsqueda de la verdad científica ${ }^{33}$.

En segundo lugar, es importante destacar sus apreciaciones en torno al binomio ciencia y democracia, donde analiza cómo esa realidad actual de la ciencia - voluble e incierta - se relaciona con la organización característica de la sociedad industrial postmoderna y el rol que en todo ello posee el mercado como variable que figura y desfigura las decisiones de la sociedad y de la autoridad ${ }^{34}$. Respecto de dicho binomio, el autor es partidario de que la búsqueda de nuevo conocimiento científico debe tener como base el respeto a los principios éticos que forman parte de una sociedad democrática, de tal manera que dicha búsqueda redunde en un mayor progreso social. Sin embargo, es en el plano de la tecnología donde el mercado concurre con mayor fuerza a modelar este progreso, siendo muchas veces los "aventureros del capital” quieres tiran de él a pesar de las incontables vicisitudes que puede sufrir la economía ${ }^{35}$.

De lo anterior nacen las dos grandes advertencias que formula el autor hacia el final de su ensayo. La primera, que, a través de la ciencia y la tecnología, el hombre recibe información como nunca antes lo había percatado, empoderándolo aún más en el proceso de toma de decisiones, cuestión que solo irá en aumento en los próximos años ${ }^{36}$. La segunda, que esta suma de decisiones crea tendencias espontáneas, las cuales resultan ser modeladas por ciertos valores culturales y, en último caso, por la propia autorregulación social ${ }^{37}$. Ello lo lleva a sostener que el producto de la ciencia hace tiempo dejó de ser propiedad

Íbid., p. 183.

Íbid., p. 186.

Íbid., pp. 196-197.

Íbid., p. 218.

Íbid., p. 220.

Íbid., p. 229.

Íbid., p. 230.

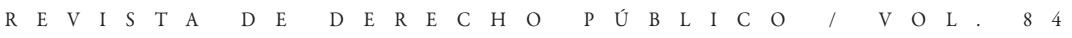


de la misma, pasando a ser de variados sectores representativos de la sociedad, como universidades, centros de investigación, organizaciones no gubernamentales, etc. En virtud de ello, el progreso social demanda que estas entidades y el resto de los actores sociales conscientes del carro de la evolución, tiren de él con la fuerza justa y necesaria para arrastrar no sólo a quienes representan sino a todos los actores de la sociedad. Aquí radicaría, entonces, la primacía del principio democrático.

\section{Algunos comentarios finales}

La obra, sin duda, tiene varias virtudes. En primer lugar, es breve y de fácil lectura. Desde sus primeras páginas el autor principal plantea el tema de una manera cautivante. El lector podrá entender fácilmente el porqué del título elegido y la transcendencia que tiene hablar de la ciencia como un nuevo poder. Luego, la obra es completa. Revisa de manera global la mayoría de los tópicos relacionados con la influencia de la ciencia en el Derecho Público y, en particular, en el Derecho Administrativo: gobernanza, labor de la administración pública, colaboración privada, normativas técnicas, actividad de certificación, agencias reguladoras, principio de precaución, tratamiento de recursos naturales, seguridad alimentaria, regulación sanitaria, entre varias otras. En este sentido, a la obra le sobra realidad, siendo un punto a agradecer por la comunidad jurídica. Finalmente, la obra es reveladora. No solo porque vuelve a poner sobre la mesa a la gestión de los riesgos como mecanismo frente al cual el Derecho Administrativo debe poner su máxima atención, sino porque brinda luces claras sobre los desafíos actuales y futuros respecto de los cuales urge una real preocupación, tanto de los gobernantes como de la sociedad en general.

Ambos autores, desde el derecho y desde la ciencia, nos entregan sus mejores esfuerzos al bosquejar el camino por el cual transita el Derecho Público en el siglo XXI. Muy probablemente, hacia el futuro gozaremos de nuevos y lúcidos aportes como este. Lo cierto, claro está, es que las preguntas que genera la entrada de la ciencia en el ámbito del derecho recién comienzan. A ellas debemos estar permanentemente atentos. 